\title{
Revisiting Euler's Orbital Calculations for the Comet of 1742
}

\author{
Sylvio R. Bistafa \\ University of São Paulo, São Paulo, Brazil \\ Email:sbistafa@usp.br
}

How to cite this paper: Bistafa, S. R. (2021). Revisiting Euler's Orbital Calculations for the Comet of 1742. Advances in Historical Studies, 10, 73-92. https://doi.org/10.4236/ahs.2021.101007

Received: January 8, 2021

Accepted: March 22, 2021

Published: March 25, 2021

Copyright $\odot 2021$ by author(s) and Scientific Research Publishing Inc. This work is licensed under the Creative Commons Attribution International License (CC BY 4.0).

http://creativecommons.org/licenses/by/4.0/

\begin{abstract}
The calculations made by Euler for the orbital parameters for the Comet of 1742 are revisited. These calculations were published in Latin in E58-Determinatio orbitae cometae qui mense Martio huius anni 1742 potissimum fuit observatus (Determination of the orbit of the comet which was clearly observed in the month of March of 1742). The present work begins by giving some background on comets before addressing the main topic itself, which consists on the calculation of the orbital parameters for the assumed parabolic and elliptical orbit made by Euler for the Comet, making use of three observations. Because of the extent of the work, characterized by rather lengthy and repetitive calculations, only the parabolic orbit case is considered here. More specifically, the work addresses the calculations for the standard parameters: longitude of the ascending node, inclination of the orbit, argument of the perihelion, the perihelion distance, which, together with the time that the comet is at the perihelion, make five unknowns that fully characterize the parabolic orbit of a comet.
\end{abstract}

\section{Keywords}

Euler's Works on Astronomy, History of Orbital Calculations, Investigations on Comets Orbits, Astronomical Calculations, Astronomical Observations

\section{Introduction}

Figure 1 shows the orbit of Halley's Comet and its predicted location in 2024 relative to the orbits of the planets. Blue is above the plane of the ecliptic and green is below. Almost the entire Halley orbit is below the plane of the ecliptic. Further, Halley revolves around its orbit in retrograde motion (the opposite sense from planet revolution). In the preceding view, the planets revolve coun- 
ter-clockwise and Halley revolves clockwise. Figure 2 shows the same thing, but from a top view.

Figure 3 shows the solar system view from the plane of the ecliptic. This is an imaginary plane used as the reference plane for the solar system. Most planets in our solar system orbit the Sun on or near this plane. The Earth's rotational axis is inclined at almost 23.5 degrees with respect to the perpendicular of the ecliptic plane. This inclination is what produces the seasons on the Earth.

Figure 4 shows the ecliptic coordinate system with the reference direction in the vernal equinox $(\Upsilon)$. The coordinates are celestial (or ecliptic) longitude $(\lambda)$ and celestial (or ecliptic) latitude ( $\beta$ ).

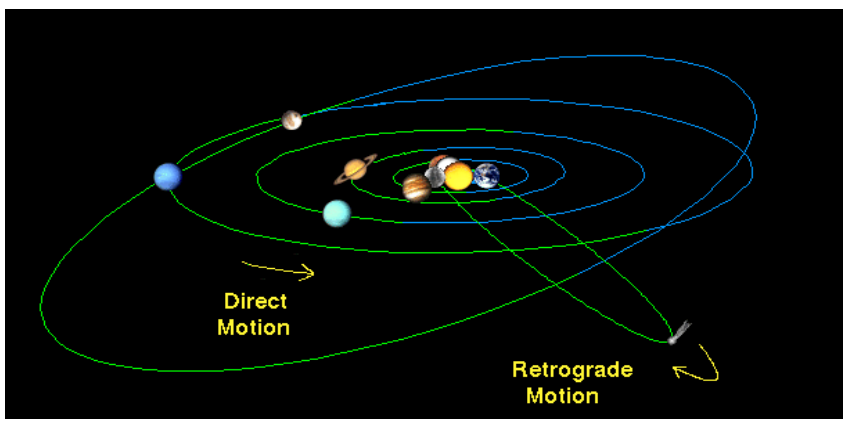

Figure 1. Halley's comet in 2024. Source:

http://www.pas.rochester.edu/ blackman/ast104/halley.html.

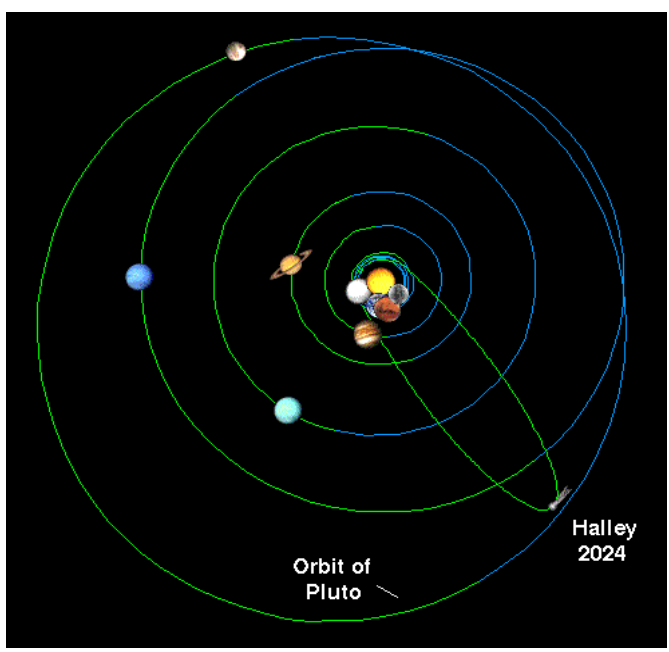

Figure 2. Halley's comet in 2024-top view. Source: http://www.pas.rochester.edu/ blackman/ast104/halley.html.

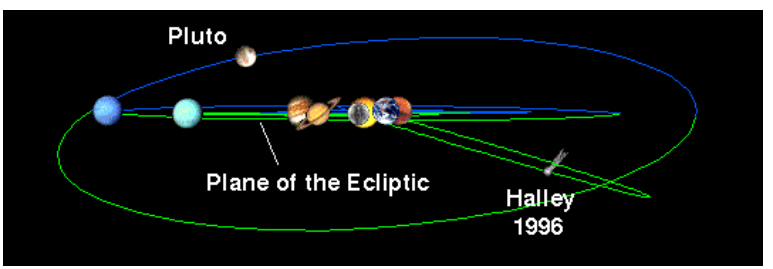

Figure 3. Solar system view from the Ecliptic plane. Source: http://www.pas.rochester.edu/ blackman/ast104/halley.html. 


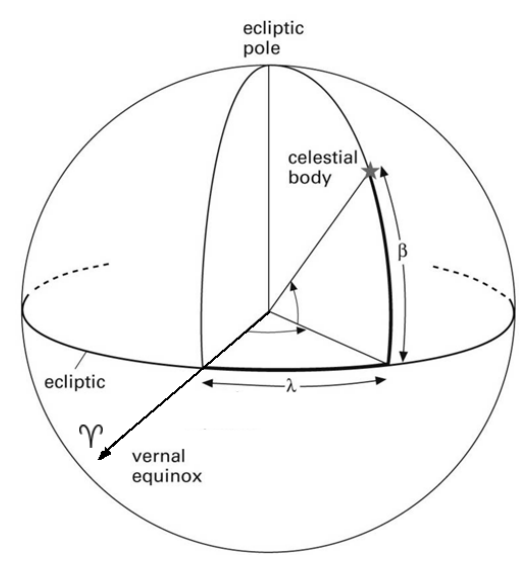

Figure 4. The ecliptic coordinate system.

The celestial latitude $(\beta)$ of a star, etc., is its angular distance (from $0^{\circ}$ to $90^{\circ}$ ) north (counted positive) or south (counted negative) of the ecliptic; it is measured along the great circle through the body and the poles of the ecliptic. The celestial longitude $(\lambda)$ of a body is its angular distance (from $0^{\circ}$ to $360^{\circ}$ ) from the vernal equinox, measured eastward along the ecliptic to the intersection of the body's circle of longitude; it is measured in the same direction as the Sun's apparent annual motion. Although observations are taken from the Earth's surface the coordinates should strictly be geocentric and, tabulated as such, are universally applicable. A slight correction is therefore applied to convert surface (topocentric) observations to geocentric values.

Figure 5 shows the trajectory of Comet Y4 ATLAS. Discovered by the ATLAS (Asteroid Terrestrial-impact Last Alert System) survey on the evening of December 28th, 2019, Comet Y4 ATLAS was the last comet discovery of 2019. Though it was only at magnitude +19.6 at the time of discovery ${ }^{1}$, the orbit of Y4 ATLAS is intriguing, bringing it in just $0.262 \mathrm{AU}$ (39.2 million kilometers or 24.4 million miles) from the Sun in late May 2020. That's interior to Mercury at perihelion, at $0.307 \mathrm{AU}$ from the Sun. This comet also grabbed observer's attention in January 2020 when it jumped 5 magnitudes to +12 , or 100 -fold in brightness.

Figure 6 shows a comet in a parabolic orbit and the associated orbital elements. Together with $T$, the time that the comet is at the perihelion, the longitude of the ascending node $(\Omega)$, the inclination of the orbit $(i)$, the argument of the perihelion $(\omega)$, and the perihelion distance (a), make five unknowns that fully characterize the parabolic orbit of a comet. As regards to $i$, if one stands at the ascending

${ }^{1}$ The visual brightness of comets is measured by the visual magnitude scale. It can sometimes be referred to as the "apparent" magnitude scale as it is always based on an object's brightness as viewed from the Earth. This is the same scale that is used for all astronomical objects from asteroids and meteors to planets, moons, galaxies and spacecraft. There are a few peculiar characteristics of the visual magnitude scale. The first thing that may seem a little odd for a scale is that the lower the number, the brighter the object is. Compared to many other measuring scales, this seems backward. The second characteristic is that each increase in integer represents an increase in brightness of 2.5 times. For example, an object of magnitude 5 is two and a half times the brightness of an object of magnitude 6. Source: http://www.cometwatch.co.uk/comet-info/the-magnitude-scale/. 


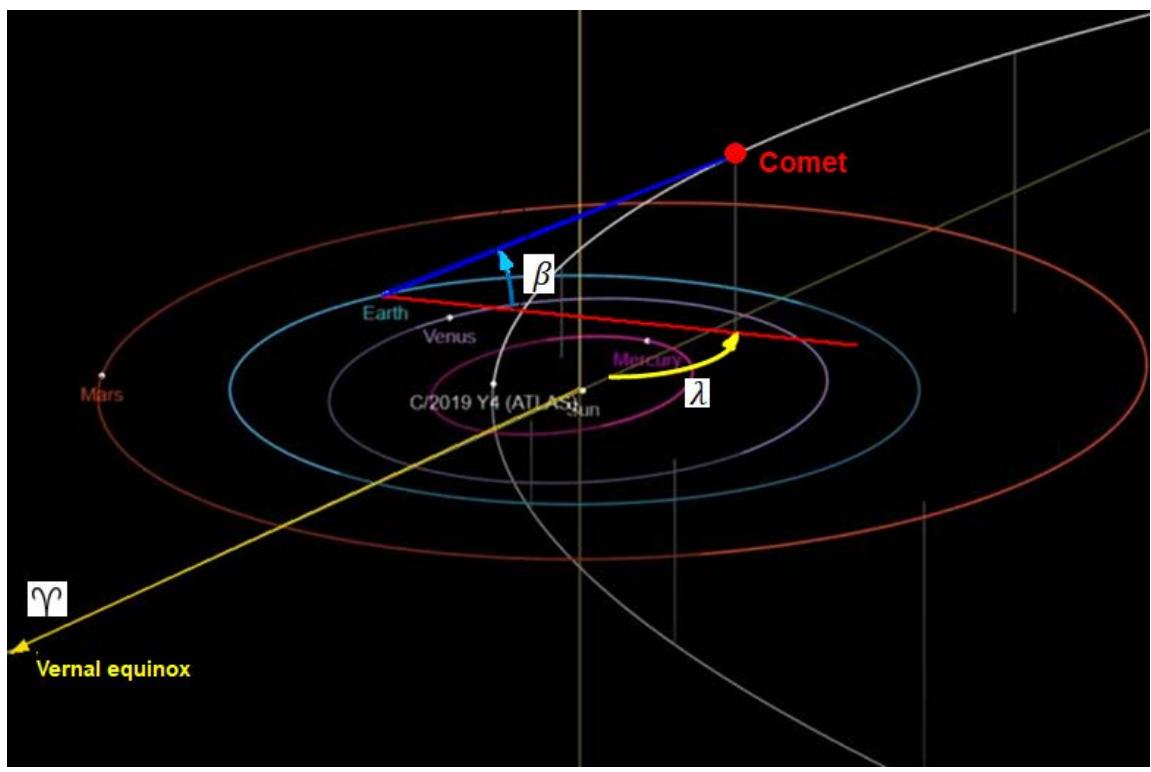

Figure 5. The orbit of Comet C/2019 Y4 ATLAS, shown near perihelion, with indications of the ecliptic geocentric longitude $(\lambda)$ and the ecliptic geocentric latitude $(\beta)$. Credit: NASA/JPL. Adapted from:

https://www.universetoday.com/145036/comet-y4-atlas-in-outburst-first-good-comet-for $\underline{-2020 / .}$

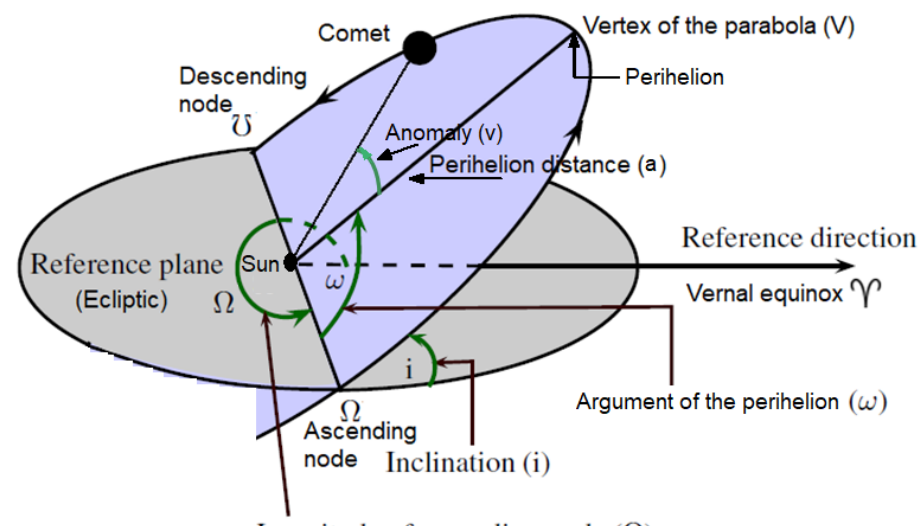

Longitude of ascending node $(\Omega)$

Figure 6. Comet in a parabolic orbit and associated orbital elements.

node and looking towards the Sun, it is reckoned upward from the plane of the ecliptic, towards the left, in the same way as angles are ordinarily reckoned. It may be anything from $0^{\circ}$ to $180^{\circ}$. If it is between $0^{\circ}$ and $90^{\circ}$, it shows that the comet is moving around the Sun in the same direction as the Earth and other planets, or that its motion is direct; if between $90^{\circ}$ and $180^{\circ}$, the comet moves in the opposite direction, or retrograde.

Now let $r$ be the distance of a comet from the Sun, and $v$ its velocity. It can be shown the if $v>h \sqrt{\frac{2}{r}}$, the comet is moving in a hyperbola; if $v=h \sqrt{\frac{2}{r}}$, its path is a parabola; and if $v<h \sqrt{\frac{2}{r}}$, it travels in an ellipse. Here $h$ is Gauss' gra- 
vitational constant, which is equal to 0.01720209895 .

Therefore, it appears that of an infinite number of velocities a comet might have, only one would cause it to move in a parabola, and so, the chances are infinity to one that no comet moves in that curve. It is, moreover, known from observation that of all comets whose orbits have been computed, not more than three of four appeared to be hyperbolas, and those were not certainly so, there is strong reason to believe that all comets are moving in ellipses. However, in the cases of all but a few their orbits are so nearly parabolas that their performances can be very well predicted by assuming a parabola as the true curve, and in the cases of a newly discovered comet, this always done (Truman, 1912). This is one of the reasons why we are only concerned here with Euler's parabolic case.

\section{Brief History of Comets Orbital Calculations}

Orbit determination has a long history, beginning with the prehistoric discovery of the planets and subsequent attempts to predict their motions. Johannes Kepler used Tycho Brahe's careful observations of Mars to deduce the elliptical shape of its orbit and its orientation in space, deriving his three laws of planetary motion in the process. The mathematical methods for orbit determination originated with the publication in 1687 of the first edition of Newton's Principia, which gave a geometrical method for finding the orbit of a body following a parabolic path from three observations. Newton's method was considered difficult to apply in practice, but it was used, with minor adaptations, by Edmund Halley to establish the orbits of various comets, including that which bears his name. The first analytical method based on three observations, that did not make use of geometrical considerations was presented by Euler in Theoria motuum planetarum et cometarum (Euler, 1744), and applied to the investigations of the comets of 1680 and 1744. This work was in turn generalized to elliptical and hyperbolic orbits by Lambert in 1761-1777.

In the years that followed, other leading mathematicians and astronomers devoted themselves to the problem. In 1774, Boscovich developed a method that also made use of three observations, published under the title De orbitus cometarum determinandis (Boscovich, 1774). In 1780, Laplace (1784) gave an entirely new approach to the problem. In contrast to others previously methods, requiring three observations relatively close to each other, Laplace's method could make use of observations separated by about 30 to 40 degrees of each other. The precision of the method improves if, indeed, it uses a more extensive set of observations, but, sometimes it is unsatisfactory when only three observations are available. Orbit determination by three observations was also employed in 1782 by the Portuguese astronomer José Monteiro da Rocha, in a method which is essentially the same as the method proposed by Olbers and published under von Zach's sponsorship two years before, in 1797 (Figueiredo \& Fernandes, 2020).

Another milestone in orbit determination was Carl Friedrich Gauss' assistance in the "recovery" of the dwarf planet Ceres in 1801. Gauss's method was able to 
use just three observations (in the form of celestial coordinates) to find the six orbital elements that completely describe an orbit.

The problem of comet's orbit determination was a recurrent one along the years that followed, such that the theory of orbit determination has been developed to the point where today it is applied in GPS receivers as well as the tracking and cataloguing of newly observed minor planets.

\section{Euler's Geometrical Calculations of the Parabolic Orbit for the Comet of 1742 (Euler, 1743)}

The geometrical method, based on three observations, was used by Euler for the investigation of the comet of 1742 (Euler, 1743). As we shall see, crucial to the developments, is the determination of the comet's distance to the Earth at the moment of the second observation, which Euler obtained by trial and error.

The mathematical formulation for the calculation of the orbital parameters is essentially in plane geometry, which, for a phenomenon that occurs in space, is rather difficult to follow from sketches of the space geometry projected in the plane of the paper. As pointed out earlier, the geometrical method is difficult to apply in practice. Moreover, Euler's calculations are rather cumbersome by transforming the original operations into logarithmic operations, in which all minute details are presented. By considering different conic sections for the orbit, such as parabolas, ellipses and hyperboles, the calculations turned out very repetitive and tedious. This is another reason why only the parabolic orbit case will be considered here for the comet of 1742 .

Only the paragraphs directly concerned with the parabolic case will be reviewed, and even so, they were abridged to what is real necessary to logically follow Euler's calculations. The paragraphs sub-headings in what follows are those that appear in the original publication.

Euler begins by praising Newton's theory on the motion of planets, and by commenting comets' observations and the theory of comets. Then follows a discussion on the modeling of comets' orbits from observations, by means of conics with the Sun at the focus, and by applying the principle of equal areas in equal times. He then asserts the objective of the investigation of developing a procedure to find the classical parameters that characterizes the orbit of a comet from three observations, with a final goal of checking his method by comparison of the calculated geocentric longitude and latitude of the Comet with a fourth observation.

In $\S .6,7$, and 8 Euler develops the expression for the calculation of the true anomaly of the comet in a parabolic orbit ${ }^{2}$ as

$$
t+\frac{1}{3} t^{3}=\frac{n}{N}
$$

${ }^{2}$ The same developments appear in another publication by Euler namely E840-De motu cometarum in orbitis parabolicis, solem in foco habentibus. A translation of this manuscript can be found at: https://scholarlycommons.pacific.edu/euler-works/840/. 
where $t=\tan \frac{1}{2} v, v$ is the anomaly, $n$ is the number of days that it takes for the comet to go from the perihelion $P$ to $M, N=\frac{a \sqrt{a}}{384651.5}$, and $a$ is the perihelion-Sun distance $P S$. In Figure 7, $v=P \hat{S} M$.

Euler then provides a table at the end of the manuscript that allows the direct determination of $v$ from tabulated values of the polynomial $t+\frac{1}{3} t^{3}$, obtained from the ratio $\frac{n}{N}$.

The paragraphs that follow, relate to the calculation of the parameters that fully characterize the parabolic orbit of the Comet of 1742 .

\section{§. XXIII.}

Besides the observations on Mar. $3^{\mathrm{d}}, 15^{\mathrm{h}}, 10^{\prime}$, let be chosen two other observations held before and after the conjunction ${ }^{3}$, which are not too close and not too far apart from each other. If they are chosen very close to one another, the aberration error ${ }^{4}$ may be sensible: if however, they were chosen rather far apart, they bring forth the hindrance of curvature. From these considerations, the three sequence of the Comet's position shown in Table 1 are seen most suitable for this purpose.

The time elapsed out between the first and the second observation is $3^{\mathrm{d}}, 8^{\mathrm{h}}, 21^{\prime}$ $=3.34791$ days, which is set $=m$. Indeed, the time elapsed out between the second and the third observation is $2^{\mathrm{d}}, 23^{\mathrm{h}}, 5^{\prime}=2.96180$ days, which is set $=n$, and hence, the time elapsed out between the first and the last observation $=m+$ $n=6.30971$ days.

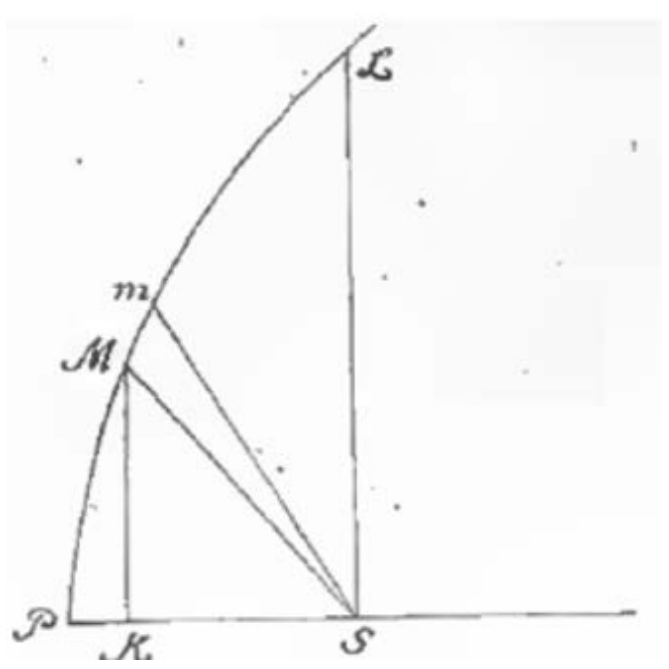

Figure 7. Geometrical elements used by Euler for the calculation of the comet's anomaly $v=P \hat{S} M$. Adapted from Figure 1 (Euler, 1743).

\footnotetext{
${ }^{3}$ In astronomy, a conjunction occurs when any two astronomical objects (such as comets, asteroids, moons, planets, and stars) appear to be close together in the sky, as observed from Earth. If two objects have the same ecliptic longitude, they are considered to be in conjunction with one another. The conjunction under consideration here is that of the Comet and the Sun as seen from the Earth. ${ }^{4} \mathrm{~A}$ small periodical change of position in the stars and other heavenly bodies, due to the combined effect of the motion of light and the motion of the observer.
} 
Table 1. Observations made by the astronomer Delisle ${ }^{5}$ for the comet of $1742^{6}$.

\begin{tabular}{|c|c|c|c|c|}
\hline Time & $\begin{array}{c}\text { Comet's [Geocentric] } \\
\text { Longitude }^{7}\end{array}$ & $\begin{array}{c}\text { Comet's [Geocentric] } \\
\text { Latitude }\end{array}$ & $\begin{array}{c}\text { Solar } \\
\text { Longitude }^{8}\end{array}$ & $\begin{array}{l}\text { Earth-Sun } \\
\text { Distance }^{9}\end{array}$ \\
\hline Feb. $28^{\mathrm{d}}, 6^{\mathrm{h}}, 49^{\prime}$ & $10^{\mathrm{S}}, 9^{\circ}, 3^{\prime}$ & $70^{\circ}, 30^{\prime}$ & $11^{\mathrm{S}}, 21^{\circ}, 4^{\prime}, 33^{\prime \prime}$ & $99,482.70$ \\
\hline${ }^{10}$ Mar. $3^{\mathrm{d}}, 15^{\mathrm{h}}, 10^{\prime}$ & $11^{\mathrm{s}}, 24^{\circ}, 24^{\prime}, 26^{\prime \prime}$ & $79^{\circ}, 31^{\prime}$ & $11^{\mathrm{S}}, 24^{\circ}, 24^{\prime}, 26^{\prime \prime}$ & $99,576.55$ \\
\hline Mar. $6^{\mathrm{d}}, 14^{\mathrm{h}}, 15^{\prime}$ & $1^{\mathrm{S}}, 12^{\circ}, 44^{\prime}$ & $77^{\circ}, 37^{\prime}$ & $11^{\mathrm{s}}, 27^{\circ}, 20^{\prime}, 48^{\prime \prime}$ & $99,660.46$ \\
\hline
\end{tabular}

\section{§. XXIV.}

Figure 8 now represents the plane of the ecliptic, in which the Sun occupies the position $S$, and the three locations of the Earth are indicated by $A, B$ and $C$ according to these three observations, and once guided by the straight lines $A S$, $B S, C S$, these segments will have the following dimensions:

$$
\begin{aligned}
& A S=99482.70 \\
& B S=99576.55 \\
& C S=99660.46
\end{aligned}
$$

and

$$
\begin{gathered}
\text { angle } A S B=3^{\circ}, 19^{\prime}, 53^{\prime \prime} \\
\text { angle } B S C=2^{\circ}, 56^{\prime}, 22 " \\
\text { angle } A S C=6^{\circ}, 16^{\prime}, 15^{\prime \prime} .{ }^{11}
\end{gathered}
$$

Now that the segment $B S$ shows the distance of the Comet to the Sun in the intermediate observation, may be drawn the lines $A \alpha$, and $C \gamma$ which designate the Comet's distances to the Earth in the first and last observation, whose positions are to be determined such that

$$
\begin{aligned}
& \text { angle } S A \alpha=42^{\circ}, 1^{\prime}, \text { angle } A m B=45^{\circ}, 20^{\prime}, 53^{\prime \prime} \\
& \text { angle } S C \gamma=45^{\circ}, 24^{\prime}, \text { angle } C n B=48^{\circ}, 20^{\prime}, 22^{\prime \prime}
\end{aligned}
$$

seconds were disregarded in the location of the Comet, because these observations are not exact beyond minutes. Then, consequently, the segments $A \alpha \& C \gamma$ ${ }^{5}$ Joseph Nicholas Delisle (1688-1768), French astronomer who graduated and later taught mathematics and astronomy at the College de France. From 1710 to 1715, he set up an observatory on the Palais de Luxembourg. In 1725 he went to St. Petersburg, where he stayed for 21 years. He was a member of the Imperial Academy of Sciences as well as director of the St. Petersburg Observatory. ${ }^{6}$ These observations were personally communicated to Euler by Delisle who took them in the St. Petersburg Observatory. Coincidentally, 1742 is the year of the death of the British astronomer Edmund Halley.

${ }^{7} 1$ Signe $\left(1^{\mathrm{s}}\right)=>30^{\circ}$. From antiquity through the 18th century, ecliptic longitude was commonly measured using twelve zodiacal signs, each of $30^{\circ}$ longitude, a practice that continues in modern astrology. The signs approximately corresponded to the constellations crossed by the ecliptic.

${ }^{8}$ Solar longitude (the ecliptic longitude of the Sun) in effect describes the position of the earth in its orbit, being zero at the moment of the vernal equinox. Solar longitude calculator https://kikimoreau.shinyapps.io/SollongCalc/.

${ }^{9}$ Roughly, the distance from Earth to the Sun is equal to about 150 million kilometers (93 million miles), which is called Astronomical Unit (AU).

${ }^{10}$ This is the conjunction time, which was not actually observed, but was estimated by Euler instead.

${ }^{11}$ These results come from the angular differences in the solar longitude for the different observations shown in Table 1. 


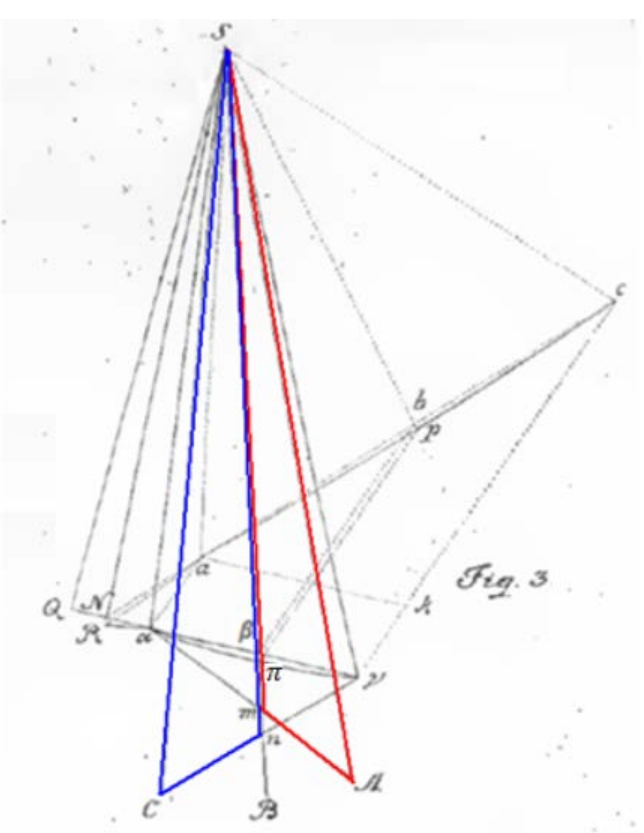

Figure 8. Triangles involved in the calculations considered in $₫$. XXIV. Adapted from Figure 3 (Euler, 1743).

intercepts the segment $B S$ in the points $m \& n$, which should be firstly defined from the following formulas, which result from the resolution of the triangles ${ }^{12}$

$$
\begin{gathered}
S m=\frac{A S \cdot \sin S A \propto}{\sin A m B}=93603.40 ; A m=\frac{A S \cdot \sin A S B}{\sin A m B}=8126.40 \\
S n=\frac{C S \cdot \sin S C \gamma}{\sin C n B}=94982.25 ; \quad C n=\frac{C S \cdot \sin B S C}{\sin C n B}=6840.66 \\
B m=B S-S m=99576.55-93603.40=5973.15 \\
B n=B S-S n=99576.55-94982.25=4594.30
\end{gathered}
$$

\section{S. XXV.}

If we now set apc as the portion of the Comet's orbit described during the interval of the assumed observations, then a will be its position in the first observation, from which the perpendicular a $a$ is dropped onto the ecliptic plane, defining the segment $A \alpha$ on this plane; and the same with $c$, which is the position of the Comet in the third observation, then, defining the segment $C \gamma$ on the ecliptic; and also $p$ is the location in the intermediate observation, with the perpendicular $p \pi$ falling onto the point $\pi$ on the line $B S$. On account that the areas described around the Sun are proportional to the times, then the ratio of area $a S p$ to the area $p S c$ is equal to the ratio of $m$ to $n$ : and if this ratio holds for the Comet's orbit projected onto the plane of the ecliptic $\alpha \pi \gamma$, then, also the ratio of the area $\alpha S \pi$ to the area $\gamma S \pi$ is as $m$ to $n$. Moreover, it is also clear that the ratio of the sectors $\alpha S \pi$ and $\gamma S \pi$ come near to approach the ratio of the triangles $\alpha S \beta \&$ $\beta S \gamma$, such that the error is certainly imperceptible: since the ratio between these

${ }^{12}$ These expressions come from the application of The Law of Sines to the triangles indicated in Figure 8. 
areas is almost the same, then because the angles $\alpha S \beta$ and $\beta S \gamma$, as the following will show, are rather small to such an extent for the curvature not to upset in any way this convenience. Then, since the ratio of the triangles $\alpha S \beta$ and $\beta S \gamma$ will be as $m$ to $n$, also $\alpha \beta: \beta \gamma=m: n$, an thus, the ratio of the segments of the cords $\alpha \gamma$ will be known by means of factors of $S B$ : whence, if the point $\beta$ in the line $B S$ becomes known, through which the cord $\alpha \gamma$ transits, at the same time, the position of the cord or angle $B \beta \gamma$ can be defined. So, let us set the angle $B \beta \gamma=\varphi$, then, in the triangle $\alpha m \beta$, the angle $\alpha m \beta=A m B \quad \& m \alpha \beta=\varphi-A m B$; in the triangle $\beta n \gamma$, indeed the angle $\beta n \gamma=C n B, \& \beta n \gamma=180^{\circ}-\varphi-B n C$. Then, it follows that ${ }^{13}$

$$
\alpha \beta=\frac{\beta m \cdot \sin A m B}{\sin (\varphi-A m B)} \text { and } \beta \gamma=\frac{\beta n \cdot \sin B n C}{\sin (\varphi+B n C)}
$$

since we have that $\alpha \beta: \beta \gamma=m: n$, and because $\sin (\varphi-A m B)=\sin \varphi \cos A m B-\cos \varphi \sin A m B \quad \&$ $\sin (\varphi+B n C)=\sin \varphi \cos B n C+\cos \varphi \sin B n C$, by dividing both denominators by $\cos \varphi$, gives

$$
\frac{n \cdot \beta m}{\tan \varphi \cdot \cot A m B-1}=\frac{m \cdot \beta n}{\tan \varphi \cdot \cot B n C+1}
$$

or

$$
\tan \varphi=\frac{\frac{m}{n} \cdot \frac{\beta n}{\beta m}+1}{\frac{m}{n} \cdot \frac{\beta n}{\beta m} \cot A m B-\cot B n C}
$$

thus, from the knowledge of the point $\beta$, the position of the cord $\alpha \gamma$ becomes known, and further, from the given latitudes, the points $a$ and $c$ in the Comet's orbit.

\section{§. XXVI.}

Therefore, this matter has been reduced to the question of defining the point $\beta$ on the line $B \beta$, such that the solution of the problem lies just on one unknown. Yet, it is not fit, as is usually done in other circumstances, to employ some character to this unknown, and to determine it by an equation, because of the too complicated expressions to which we would have arrive. Then, it will be employed some tentative values for $B \beta$, indeed ruling out the false ones, such that the most problematic undefined letters are avoided in the trigonometric calculation (Figure 9).

Let then be assumed some arbitrary values for the interval $B \beta$, and for whatever convenience the angle $\varphi=B \beta \gamma$ will be defined, and two of the Comet's position $a$ and $c$ will be held, as well as their distances to the Sun $A S, C S$, and they will be able to assign both the angle $a S c$ and the cord $a c$. Then, from the developments of the preceding sections, having been established that the Comet's orbit is a parabola or an ellipse, no matter how oblong, let be theoretically ${ }^{13}$ These expressions come from the application of The Law of Sines to the triangles indicated in Figure 9, which are drawn on the ecliptic plane. 


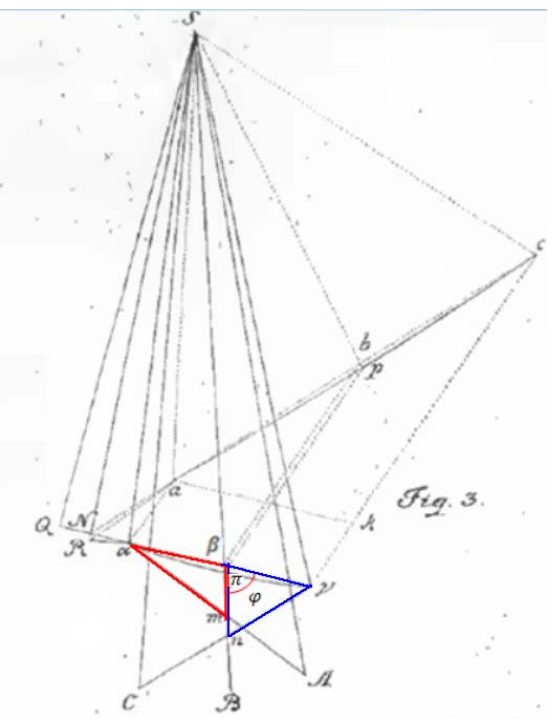

Figure 9. Triangles involved in the calculations considered in $\$$. XXVI. Adapted from Figure 3 (Euler, 1743).

found the time that the Comet should had taken to travel the distance ac, and how this time compares with the observed time of 6.30971 days, and this will be the evidence that the true value for $B \beta$ has been assumed, if however, it disagrees, the approximate true value is found from the comparison of two additional hypothesis for $B \beta$. Let then be set again two values for $B \beta$ itself, not too much different from the one that was found, such that neither of them are not too much in disagreement with the reality, for the approximate true value of $B \beta$ to be found.

\section{§. XXVII.}

Therefore, two values 8000 and 9000 will be attributed to $B \beta$ itself, since I already know from other rather crude attempts that the true value lies between these limits; and I will first determine the angle $\varphi=B \beta \gamma$.

From $₫$. XXV. We know that

$$
\varphi=\tan ^{-1}\left[\frac{\frac{m}{n} \cdot \frac{\beta n}{\beta m}+1}{\frac{m}{n} \cdot \frac{\beta n}{\beta m} \cot A m B-\cot B n C}\right]
$$

where

$$
\frac{m}{n}=\frac{3.34791}{2.96180}=1.130
$$

for

$$
B \beta=8000\left\{\begin{array}{l}
\beta m=B \beta-B m=8000-5973.15=2026.85 \\
\beta n=B \beta-B n=8000-4594.30=3405.70
\end{array} \Rightarrow \varphi=71^{\circ} 12^{\prime} 22^{\prime \prime}\right.
$$

for

$$
B \beta=9000\left\{\begin{array}{l}
\beta n=B \beta-B n=9000-4594.30=3026.85 \\
\beta m=B \beta-B m=9000-5973.15=4405.70
\end{array} \Rightarrow \varphi=74^{\circ} 27^{\prime} 29^{\prime \prime}\right.
$$


Finally

for

$$
B \beta=8000\left\{\begin{array}{l}
\alpha m \beta=A m B=45^{\circ} 20^{\prime} 53^{\prime \prime} \\
A \propto \beta=m \propto \beta=\varphi-A m B=25^{\circ} 51^{\prime} 29^{\prime \prime} \\
\beta n \gamma=C n B=48^{\circ} 20^{\prime} 22^{\prime \prime} \\
C \gamma \beta=180^{\circ}-\varphi-\beta n \gamma=60^{\circ} 27^{\prime} 16^{\prime \prime}
\end{array}\right.
$$

for

$$
B \beta=9000\left\{\begin{array}{l}
\alpha m \beta=A m B=45^{\circ} 20^{\prime} 53^{\prime \prime} \\
A \propto \beta=m \propto \beta=\varphi-A m B=29^{\circ} 6^{\prime} 36^{\prime \prime} \\
\beta n \gamma=C n B=48^{\circ} 20^{\prime} 22^{\prime \prime} \\
C \gamma \beta=180^{\circ}-\varphi-\beta n \gamma=58^{\circ} 12^{\prime} 9 "
\end{array}\right.
$$

\section{§. XXVIII.}

Now, in the triangles $\alpha m \beta, \beta m \gamma$ all angles and the sides $\beta m$ and $\beta n$ are given, and the remaining sides will be defined by the following formulas

$$
\begin{gathered}
\alpha \beta=\frac{\beta m \cdot \sin \alpha m \beta}{\sin A \alpha \beta}= \begin{cases}3305.98 & \text { for } B \beta=8000 \\
4426.15 & \text { for } B \beta=9000\end{cases} \\
\alpha m=\frac{\beta n \cdot \sin \varphi}{\sin A \alpha \beta}= \begin{cases}4399.44 & \text { for } B \beta=8000 \\
5994.33 & \text { for } B \beta=9000\end{cases} \\
\beta \gamma=\frac{\beta n \cdot \sin \beta n \gamma}{\sin C \gamma \beta}= \begin{cases}2924.70 & \text { for } B \beta=8000 \\
3827.71 & \text { for } B \beta=9000\end{cases} \\
\gamma n=\frac{\beta n \cdot \sin \varphi}{\sin C \gamma \beta}= \begin{cases}3706.04 & \text { for } B \beta=8000 \\
4994.15 & \text { for } B \beta=9000\end{cases}
\end{gathered}
$$

Having been found $\alpha m$ and $\gamma n$, then

$$
\begin{aligned}
& A \alpha=A m+\alpha m= \begin{cases}12525.84 & \text { for } B \beta=8000 \\
14120.73 & \text { for } B \beta=9000\end{cases} \\
& C \gamma=C n+n \gamma= \begin{cases}10546.70 & \text { for } B \beta=8000 \\
11834.81 & \text { for } B \beta=9000\end{cases}
\end{aligned}
$$

\section{XXIX.}

Now, from the latitudes that has been observed, the segments aa and $c \gamma$ will be obtained from $a \alpha=A \propto \cdot \tan$ lat. and $c \gamma=C \gamma \cdot \tan$ lat. (Figure 10). Indeed, the latitude in the first observation $=70^{\circ}, 30^{\prime}$, and the latitude in the third observation $=77^{\circ}, 37^{\prime}$. Then,

$$
a \alpha=A \alpha \cdot \tan 70^{\circ}, 30^{\prime}= \begin{cases}35371.88 & \text { for } B \beta=8000 \\ 39875.71 & \text { for } B \beta=9000\end{cases}
$$

and

$$
c \gamma=C \gamma \cdot \tan 77^{\circ}, 37^{\prime}= \begin{cases}48035.68 & \text { for } B \beta=8000 \\ 53902.47 & \text { for } B \beta=9000\end{cases}
$$

Additionally, having been found $a \alpha$ and $c$, may be guided the segment $a K$ parallel to $\alpha \gamma$, then 


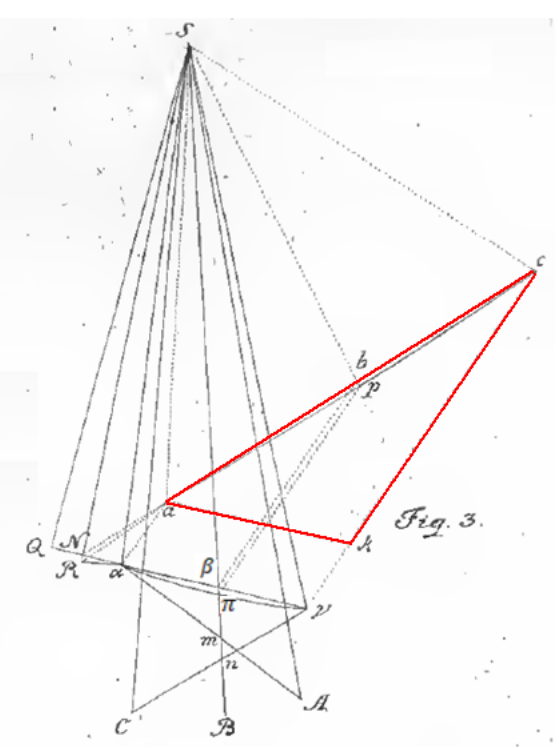

Figure 10. Triangle involved in the calculations considered in $\$$. XXIX. Adapted from Figure 3 (Euler, 1743).

$$
a K=\alpha \beta+\beta \gamma= \begin{cases}6230.68 & \text { for } B \beta=8000 \\ 8298.86 & \text { for } B \beta=9000\end{cases}
$$

and

$$
c K=c \gamma-a \alpha= \begin{cases}12663.80 & \text { for } B \beta=8000 \\ 14026.76 & \text { for } B \beta=9000\end{cases}
$$

then, since $\tan c a k=\frac{c K}{a k}$, the angle $c a k$ is found; and out of this angle, we have additionally that the cord

$$
a c=\frac{c K}{\sin c a K}= \begin{cases}14113.58 & \text { for } B \beta=8000 \\ 16297.89 & \text { for } B \beta=9000\end{cases}
$$

\section{§. XXX.}

May be guided the segments $S \alpha$ and $S \gamma$, and in the disclosed cord a $\gamma$ may be guided the normal $S Q$ : then, in the right triangle $\beta S Q$ the side $S \beta=S B-B \beta$, which together with the given angle will result in $S Q=S \beta \sin \varphi, \beta Q=S \beta \cos \varphi$ (Figure 11). Hence, $Q \alpha=Q \beta-\alpha \beta$ and $Q \gamma=Q \beta+\beta \gamma$ : and also $\frac{Q S}{Q \alpha}=\tan S \alpha Q$ and $\frac{Q S}{Q \gamma}=\tan S \gamma Q$, and once these angles are known, then, $S \alpha=\frac{Q S}{\sin S \alpha Q}$ and $S \gamma=\frac{Q S}{\sin S \gamma Q}$.

\section{§. XXXI.}

Once $S \alpha$ and $S \gamma$ are known, and since the perpendiculars a a and $c \gamma$ are given, then $\tan a S \alpha=\frac{a \alpha}{S \alpha}$ and $\tan c S \gamma=\frac{c \gamma}{S \gamma}$, producing the heliocentric longitudes $a S \alpha$ and $c S \gamma$, and then, the Comet's distances to the Sun are found out from the following formulas: 


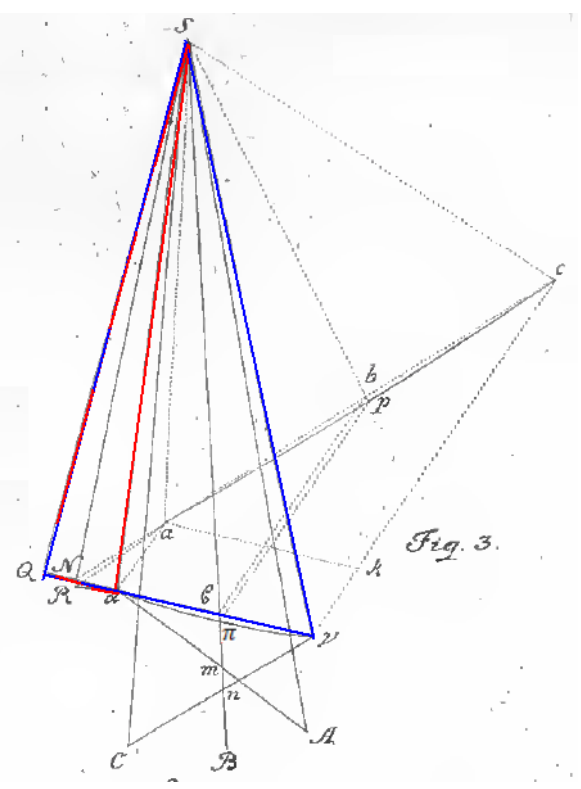

Figure 11. Triangles involved in the calculations considered in $\S$. XXX. Adapted from Figure 3 (Euler, 1743).

$$
\begin{aligned}
& S a=\frac{a \alpha}{\sin a S \alpha}= \begin{cases}97228.33 & \text { for } B \beta=8000 \\
97974.17 & \text { for } B \beta=9000\end{cases} \\
& S c=\frac{c \gamma}{\sin c S \gamma}= \begin{cases}104282.54 & \text { for } B \beta=8000 \\
106360.48 & \text { for } B \beta=9000\end{cases}
\end{aligned}
$$

\section{XXXII.}

Having been now found the two Comet's distances to the Sun $S a$ and $S c$, along with the cord $a c$, it will be possible to define the time required by the Comet to complete this distance under the hypothesis that the Comet's orbit is either parabolic or elliptic: indeed, first, if the Comet's orbit is established as a parabola, then set $S a=f, S c=g$ and $a c=k$, the time through ac will be

$$
=\frac{\left(\frac{f+g+k}{2}\right)^{3 / 2}-\left(\frac{f+g-k}{2}\right)^{3 / 2}}{3 m} \text { days }^{14} \text {, knowing that } m=384651.5, \text { which is }
$$

a constant that depends only on the Earth-Sun distance (Figure 12).

This expression gives 5.82218 days for $B \beta=8000$, and 6.70979 days for $B \beta=9000$. The actual time that the Comet took to travel to the arc ac was found to be equal to 6.30971 days. In $\$$. XXXIII, Euler found by interpolation that the value for $B \beta$ should be around 8514. Later, in $\$$. XXXVIII, Euler refined the value for $B \beta$ to 8471.61 .

\section{§. XLII.}

In this paragraph, Euler calculates the Comet's distances to the Earth in the $1^{\text {st }}$ and $3^{\text {rd }}$ observations from (Figure 13)

${ }^{14}$ This expression was developed twice by Euler, in $\S$. XIII, and in $\S$. XIV. It was also independently developed by I. H. Lambert in Insigniores Orbitae Cometarvm Proprietates (Most Remarkable Properties of Comets' Orbits), 1761, and became later known as The Euler-Lambert Equation for Parabolic Motion. 


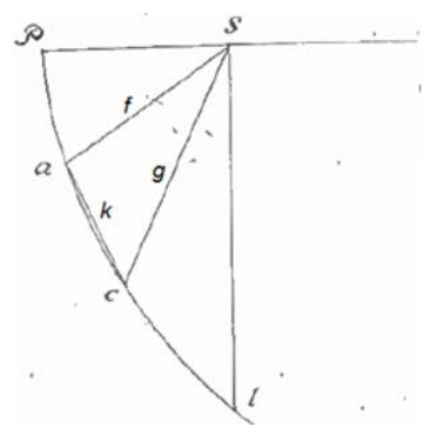

Figure 12. Geometrical elements used by Euler for the calculation of the time that it takes for the comet to travel the arc ac. Adapted from Figure 2 (Euler, 1743).

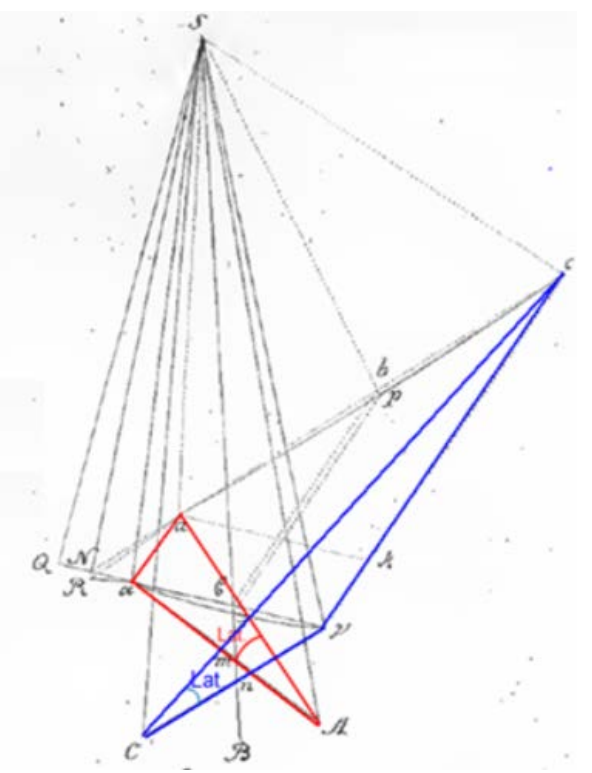

Figure 13. Triangles involved in the calculations considered in $₫$. XLII. Adapted from Figure 3 (Euler, 1743).

$$
A a=\frac{a \alpha}{\sin l a t .}=39777.5
$$

and

$$
C c=\frac{c \gamma}{\sin l a t .}=52134.6
$$

and from these, a new value for $a c=15245,34$.

\section{§. XLIII.}

When the cord ac is extended out, it meets the prolonged segment $\gamma$ a in $N$, and the point $N$ will be simultaneously in the orbit of the Comet and in the ecliptic; and if the segment $S N$ is drawn, then this segment will belong to the nodal line $\mathrm{e}^{15}$. Since the angle $a N \alpha=c a K$, then $\frac{a \alpha}{N \alpha}=\tan c a K$, and therefore,

${ }^{15}$ This happens because the nodal line is the interception of both planes, the ecliptic plane and the plane of the orbit, and then, since the cord ac belongs to the plane of the orbit, it necessarily intercepts the plane of the ecliptic in the nodal line. 
$\alpha N=\frac{a \alpha}{\tan c a K}$. Moreover, once the normal $S Q$ is drawn from $S$ to $\alpha \gamma$, the segments $S a$ and $S \gamma$ will be defined, and the angles $B S \alpha$ and $B S \gamma$ are given, the heliocentric longitude of the Comet will be obtained in the $1^{\text {st }}$ and $3^{\text {rd }}$ observation. The results are as follows:

- $\alpha N=\frac{a \alpha}{\tan c a K}$.

- Since $\beta Q=S \beta \cdot \cos \varphi$, then, $\alpha Q=\beta Q-\alpha \beta$ and $\gamma Q=\beta Q+\beta \gamma$.

- Since $S Q=S \beta \cdot \sin \varphi$, then, $S \hat{\alpha} Q=\tan ^{-1} \frac{S Q}{\alpha Q}=75^{\circ} 20^{\prime} 48^{\prime \prime}$,

$S \hat{\gamma} Q=\tan ^{-1} \frac{S Q}{\gamma Q}=70^{\circ} 59^{\prime} 48^{\prime \prime}$.

- $\alpha \hat{S} \beta=S \hat{\alpha} Q-Q \hat{\beta} S=S \hat{\alpha} Q-\varphi=2^{\circ}, 22^{\prime}, 12^{\prime \prime}$.

- Heliocentric longitude of the Comet in the $1^{\text {st }}$ observation $=$ longitude of $B+$ $\alpha \hat{S} \beta=5^{\mathrm{S}}, 24^{\circ}, 24^{\prime}, 26^{\prime \prime}+2^{\circ}, 22^{\prime}, 12^{\prime \prime}=5^{\mathrm{S}}, 26^{\circ}, 46^{\prime}, 38^{\prime \prime}$.

- $\quad \beta \hat{S} \gamma=Q \hat{\beta} S-\beta \hat{S} \gamma=\varphi-S \hat{\gamma} Q=1^{\circ}, 58^{\prime}, 48^{\prime \prime}$.

- Heliocentric longitude of the Comet in the $3^{\text {rd }}$ observation = longitude of $B$ $\beta \hat{S} \gamma=5^{\mathrm{S}}, 24^{\circ}, 24^{\prime}, 26^{\prime \prime}-1^{\circ}, 58^{\prime}, 48^{\prime \prime}=5^{\mathrm{S}}, 22^{\circ}, 25^{\prime}, 38^{\prime \prime}$.

- Heliocentric latitude of the Comet in the $1^{\text {st }}$ observation $=\tan ^{-1} \frac{a \alpha}{S \alpha}=22^{\circ}$, 36', 29".

- Sun-Comet distance in the $1^{\text {st }}$ observation $S a=\frac{a \alpha}{\sin a S \alpha}=97537.59$.

- Heliocentric latitude of the Comet in the $3^{\text {rd }}$ observation $=\tan ^{-1} \frac{C \gamma}{S \gamma}=28^{\circ}$, $55^{\prime}, 44^{\prime \prime}$.

- Sun-Comet distance in the $3^{\text {rd }}$ observation $S c=\frac{c \gamma}{\sin c S \gamma}=105270.13$.

\section{§. XLIV.}

Having been found these lines, then $Q N=\alpha Q-\alpha N$. Hence, $\tan Q S N=\frac{Q N}{Q S} ;$ therefore, $\beta \hat{S} N=\beta \hat{S} Q-Q \hat{S} N=90^{\circ}-\varphi-Q \hat{S} N=15^{\circ}, 18^{\prime}, 41^{\prime \prime}$, thus the position of the nodal line becomes known, and also the heliocentric longitude of the ascending node $N$ as

$N=\beta \hat{S S}+$ longitude of $B=15^{\circ}, 18^{\prime}, 41^{\prime \prime}+5^{\mathrm{S}}, 24^{\circ}, 24^{\prime}, 26^{\prime \prime}=6^{\mathrm{S}}, 9^{\circ}, 43^{\prime}, 7^{\prime \prime}$. T h e n , from $\alpha$, the perpendicular $\alpha R$ to $S N$ is drawn, giving $\alpha R=S \alpha \sin \alpha S R$, knowing that $\alpha \hat{S} R=Q \hat{\alpha} S-Q \hat{S} N$ and $S R=S \alpha \cos \alpha S R$; and once $a R$ is drawn, which is normal to $S R$, then it will be found out that $\tan a R \alpha=\frac{a \alpha}{\alpha R}$; the angle $a \hat{R} \alpha=\tan ^{-1} \frac{a \alpha}{\alpha R}=61^{\circ}, 43^{\prime}, 44^{\prime \prime}$ will be the inclination of the Comet's orbit in relation to the ecliptic. Hence, indeed, $a R=\frac{a \alpha}{\sin a R \alpha}$ and $\sin a S N=\frac{a R}{S a}$, and the angle $a \hat{S} N=\sin ^{-1} \frac{a R}{S a}=25^{\circ}, 52^{\prime}, 49^{\prime \prime}$ will be the heliocentric distance of the Comet to the node in the first observation. Finally, then, the angle $a \hat{S} c$ will be found from $\cos a S c=\frac{S a^{2}+S c^{2}-a c^{2}}{2 \cdot S a \cdot S c}$, and hence, the Comet's heliocentric distance to the node in the third observation

$$
\begin{aligned}
& =a \hat{S} N+a \hat{S} c=a \hat{S} N+2 \cdot \cos ^{-1} \sqrt{\left(\frac{S a+S c+a c}{2 \cdot S a}\right) \cdot\left(\frac{S a+S c-a c}{2 \cdot S c}\right)} . \\
& =25^{\circ}, 52^{\prime}, 49^{\prime \prime}+7^{\circ}, 2^{\prime}, 3^{\prime \prime}=33^{\circ}, 18^{\prime}, 52^{\prime \prime}
\end{aligned}
$$




\section{XLV.}

Having been now found both Comet-Sun distances $S a=f ; S c=g$, together with the cord $a c=k$ or the angle $a \hat{S} c=q$, it will be possible to define the parabolic and the elliptical orbit of the Comet. So, be considered the angle $P \hat{S} a=\nu$, and if it is set $B=g \cos q-f$ and $C=\frac{g-f}{1-\alpha}$, then, $\sin \frac{1}{2} v=\frac{\sqrt{(k+B)(k+C)}-\sqrt{(k-B)(k-C)}}{2 k}$, where for the parabola $\alpha=0$ and for the ellipse $\alpha=\frac{1}{50}$. Thus, having been found the angle $v$, then $P S=a=\frac{f}{2-\alpha}+\frac{f(1-\alpha)}{2-\alpha} \cos v \cdot{ }^{16}$ For the parabolic orbit, the numerical value found by Euler for the perihelion distance is $P S=a=75210.10$, and for the anomaly $a \hat{S} p=v=57^{\circ}, 10^{\prime}, 5^{\prime \prime}$. Then, by subtracting, from this angle, the angle $a \hat{S} N=25^{\circ}, 52^{\prime}, 49^{\prime \prime}$, the distance of the perihelion to the node was found as $\omega=31^{\circ}, 17^{\prime}, 16^{\prime \prime}$.

\section{§. XLVI.}

Having been found the orbit of the Comet, it remains to be defined the time in which the Comet transits by the perihelion, which will be found out, if we determine the time that it will take for the Comet to move from the perihelion $P$ to the position a. Be set $\tan a \hat{S} P=\tan \frac{1}{2} v=t$, then the time that it takes for the Comet to arrive at a from $P$, expressed in days $=\frac{a \sqrt{a}}{384651.5}\left(t+\frac{1}{3} t^{3}\right)$, and by putting $\frac{a \sqrt{a}}{384651.5}=N$, and $T=+\frac{1}{3} t^{3}$, gives the time for going through $P a=N T$. These give $T=0.598773, N=53.623, N T=32.10771=32^{\mathrm{d}}, 2^{\mathrm{h}}, 35^{\prime}$. Yet, the Comet is seen at $a$ in Feb. $28^{\mathrm{d}}, 6^{\mathrm{h}}, 49^{\prime}$, therefore, the Comet had been at the perihelion on Jan. $27^{\mathrm{d}}, 4^{\mathrm{h}}, 14^{\prime}$.

\section{\$. XLVII.}

In this paragraph, Euler gives the calculated parabolic orbital parameters to be applied to the calculations that follow in the next paragraphs.

- Distance from the perihelion to the Sun $a=75210.10$.

- Semi latus rectum $b=150420.20$.

- Date in January that the Comet transits by the perihelion: $27^{\mathrm{d}}, 4^{\mathrm{h}}, 14^{\prime}$.

- Distance of the perihelion to the node $=31^{\circ}, 17^{\prime}, 16^{\prime \prime}$.

- Heliocentric longitude of the ascending node $=6^{\mathrm{S}}, 9^{\circ}, 43^{\prime}, 7^{\prime \prime}$.

- Inclination of the Comet's orbit $=61^{\circ}, 43^{\prime}, 44^{\prime \prime}$.

\section{§. XLVIII.}

Let us calculate for the parabolic orbit case, and for the time of the last observation on March $17^{\mathrm{d}}, 7^{\mathrm{h}}, 50^{\prime}$, for which the observed longitude of the Comet was $2^{\mathrm{S}}, 18^{\circ}, 52^{\prime}$, with a northern latitude $=63^{\circ}, 13^{\prime}$. Indeed, for this time the Earth's position ${ }^{17}$ was drawn $=6^{S}, 7^{\circ}, 57^{\prime}, 30^{\prime \prime}$ and the Earth-Sun distance $=99970.05$.

${ }^{16}$ This expression was developed in $\S$. XV.

${ }^{17}$ This is actually the longitude of the Earth as seen from the Sun. 
For these conditions, and following the procedures of the previous paragraphs Euler obtained the following: true anomaly $v=74^{\circ}, 56^{\prime}, 29^{\prime \prime}$; Comet's distance to the node $=43^{\circ}, 39^{\prime}, 13^{\prime \prime}$.

\section{§. XLIX.}

Be considered in the spherical triangle $\delta c \gamma$ shown in Figure 14, the ecliptic side $\delta \gamma$, and the orbit of the Comet $\delta c$, then the angle $c \delta \gamma$ will be the inclination in relation to the ecliptic.

Then, by assuming that co is the distance from the Comet to the node, hence, $c$, indeed, will be the position of the Comet in its orbit; whence if the normal $c \gamma$ to $\delta \gamma$ is drawn, then the arc $c \gamma$ will be the heliocentric longitude of the Comet, and if $\delta \gamma$ is subtracted from the longitude of the node, the result will give the heliocentric longitude of the Comet. Indeed, from the nature of [right] spherical triangles, we have that $\sin c \gamma=\sin c \delta \cdot \sin c \delta \gamma \gamma$, and $\tan \delta \gamma=\cos c \delta \gamma \cdot \tan c \circ \delta{ }^{18}$ From these relations, the following numerical results were found by Euler: Heliocentric Latitude of the Comet $c \gamma=43^{\circ}, 39^{\prime}, 13^{\prime \prime}$; Heliocentric Longitude of the Comet $5^{\mathrm{S}}, 15^{\circ}, 24^{\prime}, 3^{\prime \prime}$.

\section{§. L.}

Then, in Figure 15 let the Sun be set at $S$ and $T$ be the position of the Earth, then, outside the plane of the ecliptic, emerges the Comet at $c$, whence in the ecliptic, falls the perpendicular $c \gamma$, and $S \gamma$ can be drawn, being the angle $c \hat{S} \gamma$ the heliocentric latitude of the Comet. Then, $c \gamma=S c \sin c S \gamma$, and $S \gamma=S c \cos c S \gamma$. Now, from $\gamma$, be drawn the perpendicular $\gamma p$ to $S T$, then, from the given angle $\gamma \hat{S} T$, which is certainly the difference between the heliocentric longitudes of the Earth and the Comet $\gamma p=S \gamma \sin \gamma S T$ and $S p=S \gamma \cos \gamma S T$, with $T p=S T-S p$. From these, it is found out that $\tan S T \gamma=\frac{p \gamma}{p T}$, which is the angle that when added to the longitude of the Sun, gives the geocentric longitude of the Comet (Figure 16).

Besides, we have that $T \gamma=\frac{p \gamma}{\sin S T \gamma}$; and $\tan c T \gamma=\frac{c \gamma}{T \gamma}$; will be the geocentric latitude of the Comet, and, finally, $\frac{c \gamma}{\sin c T \gamma}=T c$ is the distance from the Comet to the Earth.

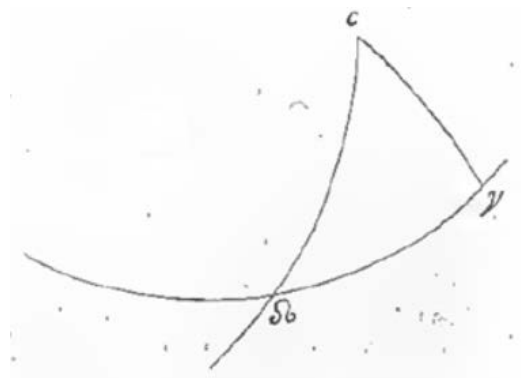

Figure 14. Spherical triangle $\delta \propto \gamma$ for the calculation of the heliocentric longitude of the Comet in the last observation. Adapted from Figure 4 (Euler, 1743).

${ }^{18}$ These relations come from the application of Napier's rules for this right spherical triangle. 


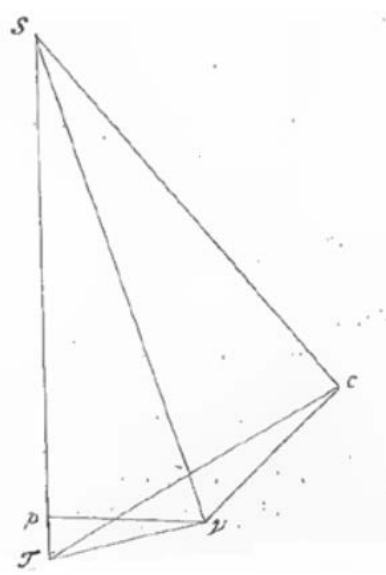

Figure 15. Auxiliary figure provided by Euler to determine the geocentric latitude and longitude of the comet. ${ }^{19}$ Adapted from Figure 5 (Euler, 1743).

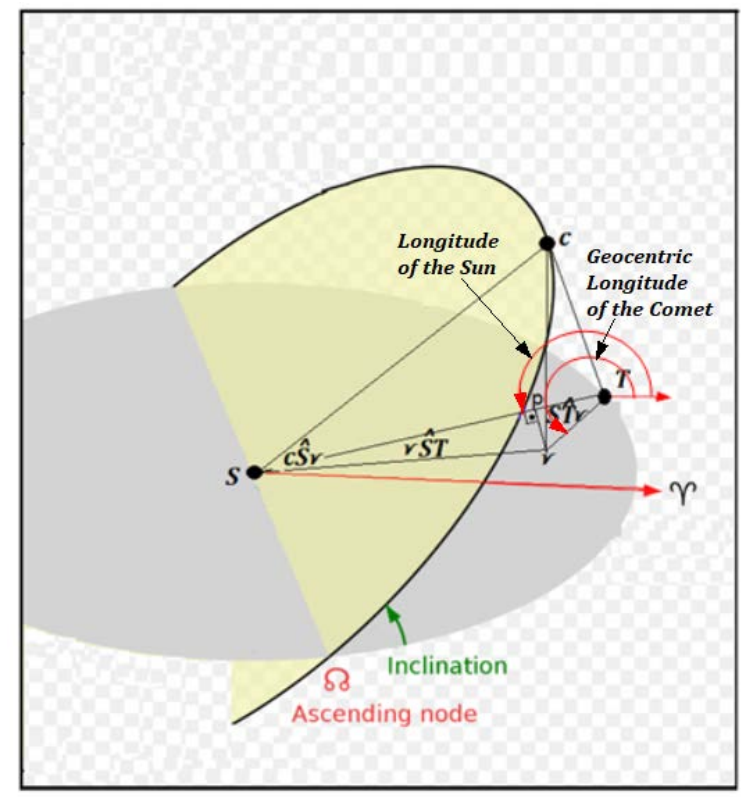

Figure 16. Geocentric longitude of the comet.

From these expressions, Euler calculates $S \hat{T} \gamma=2^{S}, 11^{\circ}, 8^{\prime}, 21^{\prime \prime}$, which when added to the longitude of the Sun $=7^{\circ}, 57^{\prime}, 30^{\prime \prime}$ $\left[\lambda_{\text {Sun }}=6^{\mathrm{S}}, 7^{\circ}, 57^{\prime}, 30^{\prime \prime}+180^{\circ}=7^{\circ}, 57^{\prime}, 30^{\prime \prime}\left(\bmod 360^{\circ}\right)\right]$, gives $2^{\mathrm{s}}, 19^{\circ}, 5^{\prime}, 51^{\prime \prime}$ for the geocentric longitude of the Comet. For the geocentric latitude of the Comet, Euler obtains $62^{\circ}, 6^{\prime}, 10^{\prime \prime}$.

In $\$$. LI, Euler compares the observed geocentric longitude of the Comet of $2^{\mathrm{S}}$, $18^{\circ}, 52^{\prime}$ with the calculated value of $2^{\mathrm{S}}, 19^{\circ}, 5^{\prime}, 51^{\prime \prime}$, saying that they do not too

${ }^{19}$ The ecliptical longitude $\lambda$ (lambda) is the position along the ecliptic, relative to the vernal equinox (so relative to the stars). If you look at the Sun from the Earth, then you're looking in exactly the opposite direction than if you look at the Earth from the Sun, so those directions are $180^{\circ}$ apart. Hence, the ecliptic longitude of the Sun, as seen from the Earth, is equal to $\lambda_{\text {Sun }}=\lambda_{\text {Earth }}+180^{\circ}$. The value of $\lambda_{\text {sun }}$ determines when the (astronomical) seasons begin: when $\lambda_{\text {sun }}=0$, then spring begins in the northern hemisphere, and autumn in the southern hemisphere. Each next multiple of $90^{\circ}$ brings the start of the next season. 
much disagree, whereas the observed geocentric latitude of $63^{\circ}, 13^{\prime}$ much disagrees with the calculated value of $62^{\circ}, 6^{\prime}, 10^{\prime \prime}$, and concludes that this disagreement can be attributed to error in the observation.

\section{Conclusion}

Euler's calculations for investigating the parabolic orbit for the Comet of 1742 were revisited. Besides the true anomaly, Euler succeeded in finding the classical orbital parameters from three observations of the Comet taken from the Earth: longitude of the ascending node, the inclination of the orbit, the argument of the perihelion, and the perihelion distance, which fully characterized the orbit of that Comet. By the end of the manuscript, Euler uses his method to compute the geocentric longitude and latitude of the Comet in the last observation, and compared them with the measurements. The geocentric longitude agreed quite well, however, the geocentric latitude showed some deviation, which Euler attributed to an error in the observation.

\section{Conflicts of Interest}

The author declares no conflicts of interest regarding the publication of this paper.

\section{References}

Boscovich, R. J. (1774). De orbitus cometarum determinandis ope trium observationem parum a se invicem remotarum. Mémoires de Mathématique et de Physique, présentes à Académie Royale des Sciences par divers Savants, 6, 198-215.

Euler, L. (1743). Determinatio orbitae cometae qui mense Martio huius anni 1742 potissimum fuit observatus. Miscellanea Berolinensia, 7, 1-90.

Euler, L. (1744). Theoria motuum planetarum et cometarum. Berlin: Ambrose Haude.

Figueiredo, F. B., \& Fernandes, J. M. (2020). José Monteiro da Rocha (1734-1819) and His 1782 Work on the Determination of Comet Orbits. Journal for the History of Astronomy, 51, 461-481. https://doi.org/10.1177/0021828620947844

Laplace, P. S. (1784). Mémoire sur la Détermination des Orbites des Comètes. Mémoires de l'Académie Royale des Sciences de Paris, 10, 93-146. https://gallica.bnf.fr/ark:/12148/bpt6k775981/f1.item

Truman, O. H. (1912). Computation of a Comet's Orbit. MS (Master of Science) thesis, Iowa: State University of Iowa. https://doi.org/10.17077/etd.c9h10h63 